\title{
WEAKLY NONLINEAR OSCILLATORY CONVECTION IN A VISCOELASTIC FLUID SATURATED POROUS MEDIUM WITH THROUGHFLOW AND TEMPERATURE MODULATION
}

\author{
P. KIRAN ${ }^{*}$ and Y. NARASIMHULU \\ Department of Mathematics, Rayalaseema University \\ Kurnool-518002, AP, INDIA \\ E-mails: kiran40p@gmail.com; ynarasimhulu@redi.mail.com \\ S.H. MANJULA \\ Department of Mathematics, Vignan's University \\ Vadlamudi, Guntur 522213, Andhra Pradesh, INDIA \\ E-mail: manjubknd.bk@gmail.com
}

\begin{abstract}
The effect of vertical throughfow and temperature modulation on a viscoelastic fluid saturated porous medium has been investigated. The amplitudes of temperature modulation at the lower and upper surfaces are considered to be very small and the disturbances are expanded in terms of power series of amplitude of convection. A weak nonlinear stability analysis has been performed for the oscillatory mode of convection, and heat transport in terms of the Nusselt number, which is governed by the non autonomous complex GinzburgLandau equation, is calculated. The effect of vertical through flow is found to stabilize the system irrespective of the direction of through flow in the case of permeable boundary conditions. The time relaxation has a destabilizing effect, while the time retardation parameter has a stabilizing effect on the system. The effects of amplitude and frequency of modulation on heat transport have been analyzed and depicted graphically. The study shows that the heat transport can be controlled effectively by a mechanism that is external to the system. Further, it is also found that heat transfer is more in oscillatory mode of convection rather than in stationary mode of convection.
\end{abstract}

Key words: throughflow, temperature modulation, weak nonlinear theory, complex Ginzburg-Landau model.

\section{Introduction}

The problem of convective instability of a horizontal fluid saturated porous layer has been investigated extensively, and is well documented by Ingham and Pop [1], Nield and Bejan [2], Vafai [3, 4]. The concept of regulating convective instabilities is an important topic in thermal and engineering sciences. Such a regulation like thermal, gravitational, rotational and magnetic field modulations etc., can be used in order to alter or regulate convection. As pointed out by Davis [5], the dynamic of stabilization and destabilization may lead to dramatic changes of behavior depending on the proper tuning of the amplitude and frequency of the modulation. If an imposed modulation can destabilize an otherwise stable state, then there can be a major enhancement of heat/mass/momentum transport. If an imposed modulation can stabilize an otherwise unstable state, then higher frequencies can be attained in various processing techniques. One of the effective mechanisms that is considered in this paper is a time periodic heating of wall temperature (thermal modulation) at the boundaries. The related problems are investigated by Venizian [6], Caltagirone [7], Chhuon and Cal-tagirone [8], Rudraiah et al. [9, 10], Rudraiah and Malashetty [11], Malashetty and

\footnotetext{
${ }^{*}$ To whom correspondence should be addressed
} 
Wadi [12], Bhatia and Bhadauria [13, 14], Bhadauria and Bhatia [15], Bhadauria [16-20], Bhadauria [21, 22], Bhadauria and Sherani [23, 24], Bhadauria and Suthar [25], Bhadauria and Srivastava [26], Bhadauria et al. [27], and Bhadauria and Kiran [28, 29, 30] for various physical models and boundary conditions.

The flow of viscoelastic fluid is of considerable importance in various areas in science, engineering and technology, such as material processing, petroleum, chemical and nuclear industries, carbon dioxide geologic sequestration, bioengineering, reservoir engineering, biorheology, geophysics, chemical and petroleum industries. Viscoelastic fluids exhibit unique patterns of instabilities such as the overstability that is not predicted or observed in Newtonian flow. Rudraiah et al. [31, 11] studied the stability of viscoelastic fluid saturated porous layer using Darcy and Brinkman models. It is found that the effect of elasticity of the fluid is to destabilize the system and that of porosity is to stabilize the same. A theoretical analysis of thermal instability in a horizontal porous layer saturated with a viscoelastic liquid is conducted by Kim et al. [32]. It is found that the overstability is a preferred mode for a certain parameter range. They further reported on the basis of the nonlinear theory that convection has the form of a supercritical and stable bifurcation independent of the values of the elastic parameters. Later on many studies have come up on thermal convection in viscoelastic fluids. Some of them are Yoon et al. [33], Malashetty et al. [34], Bertola and Cafaro [35], Sheu et al. [36, 37], Wang and Tan [38] and Malashetty et al. [39], Kumar and Bhadauria [40, 41].

However, several geophysical and technological applications involve non-isothermal flow of fluids through porous media, called through flow. Such a flow alters the basic temperature profile from linear to nonlinear with layer height, which in turn affects the stability of the system significantly. The effect of through flow on the onset of convection in a horizontal porous layer has been studied by Wooding [42], Sutton [43], Homsy and Sherwood [44], Jones and Persichetti [45]. Nield [46]. Shivakumara [47] showed that a small amount of through flow can have a destabilizing effect if the boundaries are of different types and a physical explanation for the same was given. Khalili and Shivakumara [48] investigated the effect of through flow and internal heat generation on the onset of convection in a porous medium. They showed that through flow destabilizes the system, even if the boundaries are of the same type; a result which is not true in the absence of an internal heat source. The non-Darcian effects on convective instability in a porous medium with through flow were investigated in order to account for inertia and boundary effects by Shivakumara [49], Shivakumara and Khalili [50], Khalili and Shivakumara [51]. Later on many researchers have investigated through flow effects considering different physical models, some of them are; Shivakumara and Nanjundappa [52], Shivakumara and Sureshkumar [53], Brevdo [54], Barletta et al. [55], Nield and Kuznetsov (2011), Reza and Gupta [56] and Nield and Kuznetsov (2013).

The purpose of the present work is to study the oscillatory mode of convection in a horizontal porous layer saturated by a viscoelastic fluid using complex non autonomous Ginzburg-Landau equation (Bhadauria and Kiran [29-30, 57-58]) under vertical through flow and temperature modulation effects. Since the stationary case of the problem is just as Benard-Darcy convection in a Newtonian fluid saturated porous medium, so we intend to study oscillatory mode of convection by considering a weak nonlinear analysis and investigated heat transfer across the porous medium. To the best of authors' knowledge to date no study on oscillatory convection under temperature modulation with vertical through flow is available in the literature.

\section{Mathematical formulation}

We consider an infinitely extended horizontal fluid saturated porous layer of depth 'd' as given in Fig.1. The porous layer is homogeneous and isotropic, and saturated with a viscoelastic fluid. The porous medium is heated slowly from below. Using modified Darcy's model (Alishaev [59] and Bhadauria and Kiran [29]) and employing the Boussinesq approximation for this system, the governing equations of flow are given by 


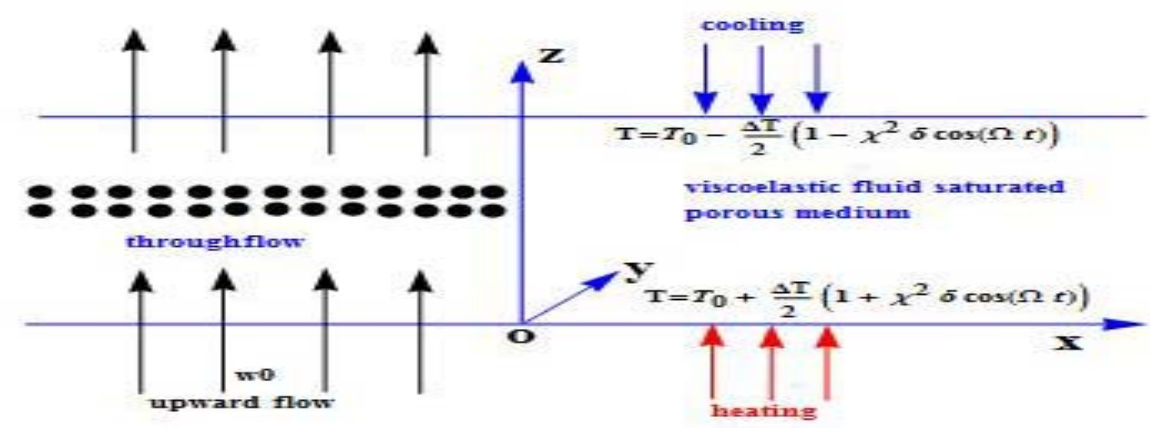

Fig.1. A sketch of the physical problem.

$\Delta . q=0$,

$\left(\bar{\lambda} \frac{\partial}{\partial t}+1\right)(-\Delta P+\rho \boldsymbol{g})-\frac{\mu}{K}\left(\bar{\varepsilon} \frac{\partial}{\partial t}+1\right) \boldsymbol{q}=0$,

$\frac{\partial T}{\partial t}+(\boldsymbol{q} \cdot \Delta) T=\mathrm{K}_{T} \Delta^{2} T, \quad \frac{\partial T}{\partial t}+(\boldsymbol{q} \cdot \Delta) T=\mathrm{K}_{T} \Delta^{2} T$,

$\rho=\rho_{0}\left[1-\alpha_{T}\left(T-T_{0}\right)\right]$

where the physical variables have their usual meaning as given in the nomenclature. The externally imposed thermal boundary conditions are considered as Venezian [6]

$$
\begin{aligned}
& T=T_{0}+\frac{\Delta T}{2}\left[1+\chi^{2} \delta_{\text {lower }} \cos (\Omega t)\right], \quad \text { at } \quad z=0, \\
& T=T_{0}-\frac{\Delta T}{2}\left[1-\chi^{2} \delta_{\text {upper }} \cos (\Omega t)\right], \quad \text { at } \quad z=d .
\end{aligned}
$$

Then we have

$$
\begin{array}{ll}
\delta_{\text {lower }}=\delta_{\text {upper }}=\delta & \text { (In-phase modulation), } \\
\delta_{\text {lower }}=-\delta_{\text {upper }}=\delta & \text { (Out-phase modulation), } \\
\delta_{\text {lower }}=\delta, \quad \delta_{\text {upper }}=0 & \text { (Lower boundary modulation) }
\end{array}
$$

where $\Delta T$ is the temperature difference across the porous medium, $\delta$ and $\Omega$ are amplitude and frequency of temperature modulation. The basic state is assumed to be quiescent and the quantities in this state are given by

$$
q_{b}=\left(0,0, w_{0}\right), \quad \rho=\rho_{b}(z, t), \quad p=p_{b}(z, t), \quad T=T_{b}(z, t) .
$$

Substituting Eq.(2.9) in Eqs (2.1)-(2.4), we get the following equations which help us in defining the hydrostatic pressure and temperature fields 


$$
\begin{aligned}
& u^{\prime}=\frac{\partial \psi}{\partial z}, \quad w^{\prime}=-\frac{\partial \psi}{\partial x}, \quad \frac{\partial p_{b}}{\partial z}=\frac{\mu}{K} w_{0}-p_{b} g \\
& \frac{\partial p_{b}}{\partial t}+w_{0} \frac{\partial T_{b}}{\partial t}=\kappa_{T} \frac{\partial^{2} T_{b}}{\partial z^{2}}, \\
& \rho_{b}=\rho_{0}\left[1-\beta_{T}\left(T_{b}-T_{0}\right)\right] .
\end{aligned}
$$

The solution of Eq.(2.11) subject to the thermal boundary conditions Eq.(2.5), is given by

$$
T_{b}(z, t)=f(z)+\chi^{2} \delta \operatorname{Re}\left[f_{l}(z, t)\right]
$$

here $f(z)$ is a steady part and $f_{l}(z ; t)$ is a oscillatory part of the basic state temperature, and will be defined later. Since the basic state is unsteady $\left[f_{l}(z, t)\right]$ and it is periodic, the repeating sequence of basic state acceleration followed by basic-state deceleration leads to ambiguities in interpretation. This basic periodic state is unstable (Rosenblat [60]) if there exists a disturbance that experiences net growth over each modulation cycle. The basic state is called transiently or monotonically stable as it is subject to a disturbance that delays or grows during part of the cycle, possibly attains an appreciable amplitude. The aggregate of many disturbances may lead (Finucane and Kelly [61]) to a flow that is continuously different from the basic state. Hence a single disturbance ultimately may decay or grow then quantities like heat $/ \mathrm{mass} / \mathrm{momentum}$ transport could differ measurably from those of the basic state. So the temperature modulation problem plays a critical role in the thermal instability problem. The finite amplitude perturbations on the basic state are superposed in the following form

$$
\boldsymbol{q}=\boldsymbol{q}_{b}+\boldsymbol{q}^{\prime}, \quad \rho=\rho_{b}+\rho^{\prime}, \quad p=p_{b}+p^{\prime}, \quad T=T_{b}+T^{\prime} .
$$

We introduce the Eq.(2.14) and the basic temperatures field in Eqs (2.1)-(2.4), and then use the stream function $\psi$ as $u^{\prime}=\frac{\partial \psi}{\partial z}, w^{\prime}=-\frac{\partial \psi}{\partial x}$, for two dimensional flows. The equations are then nondimensionalized using the physical variable; $\varepsilon=\frac{\kappa_{T} \bar{\varepsilon}}{d^{2}}, \lambda=\frac{\kappa_{T} \lambda}{d^{2}}$ and $\Omega=\frac{\kappa_{T}}{d^{2}} \Omega^{*}, \quad(x, y, z)=d\left(x^{*}, y^{*}, z^{*}\right)$, $t=\frac{d^{2}}{\kappa_{T}} t^{*}, \psi=\kappa_{T} \psi^{*}, T^{\prime}=\Delta T T^{*}$. The resulting non-dimensionalized system of equations can be expressed as (dropping the asterisk)

$$
\begin{aligned}
& \left(\varepsilon \frac{\partial}{\partial t}+1\right) \nabla^{2} \psi+\operatorname{Ra}\left(\lambda \frac{\partial}{\partial t}+1\right) \frac{\partial T}{\partial x}=0, \\
& \frac{\partial T_{b}}{\partial z} \frac{\partial \psi}{\partial x}\left(\nabla^{2}-\operatorname{Pe} \frac{\partial}{\partial z}\right) T=-\frac{\partial T}{\partial t}+\frac{\partial(\psi, T)}{\partial(x, z)} .
\end{aligned}
$$

The non-dimensionalized parameters in the above equations are: $\mathrm{Pe}=\frac{w_{0} d}{k T}$ $\frac{\partial T_{b}}{\partial z}=f^{\prime}(z)+\chi^{2} \delta \operatorname{Re}\left[f_{l}^{\prime}(z, t)\right]$. is Péclet number and $\mathrm{Ra}=\frac{\beta_{T} g \Delta T d K}{\nu k_{T}}$ is the thermal Rayleigh number. 
Equation (2.16) shows that the basic state solution influences the stability problem through the factor $\frac{\partial T_{b}}{\partial z}$, which is given by

$$
\frac{\partial T_{b}}{\partial z}=f^{\prime}(z)+\chi^{2} \delta \operatorname{Re}\left[f_{l}^{\prime}(z, t)\right]
$$

We write the nonlinear system of equations Eqs (2.15)-(2.16) in the matrix form as given below

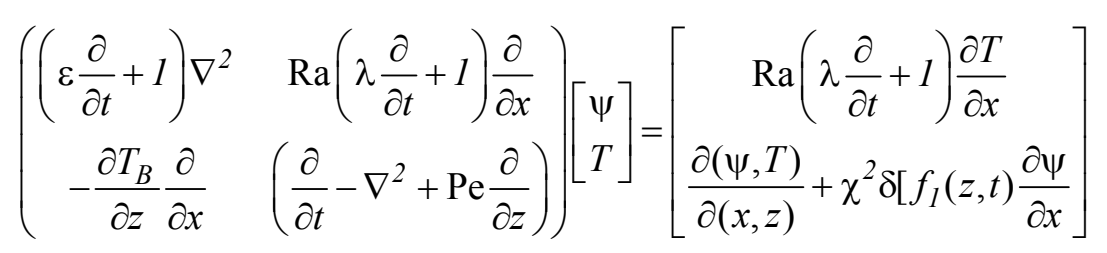

The above system will be solved by considering stress free and isothermal boundary conditions as given bellow

$$
\psi=0 \quad \text { and } \quad T=0 \quad \text { on } \quad z=0, \quad z=1 .
$$

\section{Amplitude equation for oscillatory convection}

Introduce a small perturbation parameter $\chi$ that shows deviation from the critical state of onset of convection, the variables for a weak nonlinear state may be expanded as power series of $\chi$ as

$$
\begin{aligned}
& \mathrm{Ra}=R_{0 c}+\chi^{2} R_{2}+\chi^{4} R_{4}+\ldots \ldots . . \\
& \psi=\chi \psi_{1}+\chi^{2} \psi_{2}+\chi^{3} \psi_{3}+\ldots \ldots . \\
& T=\chi T_{1}+\chi^{2} T_{2}+\chi^{3} T_{3}+\ldots \ldots .
\end{aligned}
$$

where $R_{o c}$ is the critical value of the Darcy-Rayleigh number at which the onset of convection takes place in the absence of temperature modulation. In order to allow for anticipated frequency shift along the bifurcation solution, we introduce the fast time scale of time $\tau$ and the slow time scale of $s$. Therefore, the scaling of time variable is such that $\frac{\partial}{\partial t}=\frac{\partial}{\partial \tau}+\chi^{2} \frac{\partial}{\partial s}$. In the first order problem, the nonlinear term in the energy equation will vanish. Therefore, the first order problem reduces to the linear stability problem for over stability.

\subsection{At the lowest order}

At first order we consider the following system in which the nonlinearity skipping 


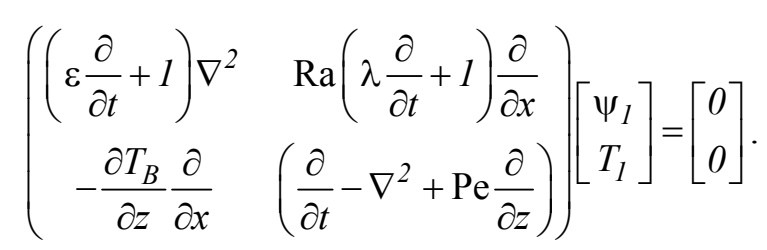

The solution of the lowest order system subject to the boundary conditions, Eq.(2.19), is assumed to be of the form

$$
\begin{aligned}
& \psi_{l}=\left(\mathbb{A}_{l}(s) e^{i \omega T}+\mathbb{A}_{l}(s) e^{-i \omega T}\right) \cos \operatorname{axsin} \pi z \\
& T_{l}=\left(\mathbb{B}_{I}(s) e^{i \omega T}+\mathbb{B}_{I}(s) e^{-i \omega T}\right) \sin \operatorname{axsin} \pi z
\end{aligned}
$$

The undetermined amplitudes are functions of the slow time scale, and are related by the following relation

$$
\text { B) }{ }_{l}(s)=\mathrm{Ra}=\frac{}{4 a^{2} \pi^{2}} \mathbb{A}_{l}(s)
$$

where $c=a^{2}+\pi^{2}$. The thermal Darcy-Rayleigh number for the stationary mode of convection can be given as

$$
\mathrm{Ra}=\frac{c^{2}\left(4 \pi^{2}+\mathrm{Pe}^{2}\right)}{4 a^{2} \pi^{2}}
$$

In the absence of through flow $(\mathrm{Pe}=0)$ we get the classical results of Horton and Rogers [62] and Lapwood [63]. We find the Darcy-Rayleigh number for oscillatory convection as given bellow

$$
\mathrm{Ra}=\frac{\left[\left(c^{2}+\omega^{2}\left(\lambda \varepsilon c^{2}+\lambda c-\varepsilon c\right)\right)\right]\left(4 \pi^{2}+\mathrm{Pe}^{2}\right)}{4 \pi^{2} a^{2}}
$$

The critical value of the wave number is defined while minimizing Ra with respect to the critical wave number. The critical value of the corresponding wave number does not depend on $(\lambda, \varepsilon)$ in the stationary mode, but in the oscillatory mode. Also, we see that the overstability can occur for a particular wave number only if the following inequality holds

$$
\lambda>\varepsilon+\frac{1}{c} .
$$

The dimensionless frequency of the neutral oscillatory mode is

$$
\omega^{2}=\frac{c(\lambda-\varepsilon)-1}{\varepsilon \lambda} \text {. }
$$

\subsection{In the second order}

In this state, we consider the nonlinear terms in the equations 


$$
\begin{aligned}
& \frac{\partial\left(\psi_{l}, T_{l}\right)}{\partial(x, z)}=\frac{\pi a}{2}\left\{\mathbb{A}_{l}(s) \mathbb{B}_{l}{ }_{l}(s) e^{2 i \omega T}+\mathbb{A}_{l}(s) \mathbb{B}_{l}{ }_{l}(s) e^{-2 i \omega T}+\mathbb{A}_{l}(s) \mathbb{B}_{l}{ }_{l}(s)+\right. \\
& \left.+\mathbb{A}_{l}(\mathrm{~s}) \mathbb{B}^{\prime}{ }_{l}(s)\right\} \sin 2 \pi z .
\end{aligned}
$$

From the above relation, we can deduce that the velocity and temperature fields have the terms having frequency $2 \omega$ and independent of the past time scale. Thus, we write the second order temperature term as follows

$$
T_{2}=\left\{T_{20}+T_{22} e^{2 i \omega \tau}+T_{22} e^{-2 i \omega \tau}\right\} \sin 2 \pi z
$$

where $T_{22}$ and $T_{20}$ are temperature fields which have the terms having frequency $2 \omega$ and independent of the fast time scale, respectively. The solutions of the second order problem are

$$
\psi_{2}=0, \quad T_{20}=\frac{a}{8 \pi}\left\{\mathbb{A}_{l}(s) \mathbb{B}_{l}(s)+\mathbb{A}_{l}(s) \mathbb{B B}_{l}(s)\right\},
$$

and

$$
T_{22}=\frac{\pi a}{8 \pi^{2}+4 i \omega} \mathbb{A}_{l}(s) \mathbb{B B}_{l}(s) .
$$

The horizontally averaged Nusselt number $\mathrm{Nu}$ for the oscillatory mode of convection is given by

$$
\mathrm{Nu}(\tau)=\frac{\text { conduction }+ \text { convection }}{\text { conduction }}
$$

Substituting the expression of Tb and T2 from Eqs (2.17)-(3.11) in Eq.(3.14) we get

$$
\mathrm{Nu}=1+\left(\frac{2 a^{2} c \pi^{2}\left(e^{\mathrm{Pe}}-1\right)}{\operatorname{Pe}\left(4 \pi^{2}+\mathrm{Pe}^{2}\right)\left(c^{2}+\omega^{2}\right)}+\frac{2 \pi^{4}\left(e^{\mathrm{Pe}}-1\right)}{\operatorname{Pe}\left(4 \pi^{2}+\mathrm{Pe}^{2}\right) \sqrt{\left(4 \pi^{4}+\omega^{2}\right)} \sqrt{\left(c^{2}+\omega^{2}\right)}}\right)\left|A_{l}(s)\right|^{2}
$$

Though our concentration is an oscillatory mode of convection, for comparison of results, we also define the Nusselt number for the stationary mode of convection as

$$
\mathrm{Nu}(\tau)=1+\frac{4 \pi^{4} k_{c}^{2}\left(e^{\mathrm{Pe}}-1\right)}{c \mathrm{Pe}\left(4 \pi^{2}+\mathrm{Pe}^{2}\right)^{2}} A_{l}^{2}(\tau)
$$

It is clear that the thermal modulation is effective at third order and affects $\mathrm{Nu}(s)$ through $A_{l}(s)$ which is evaluated at third order. We have also calculated the mean value of the Nusselt number $(\overline{\mathrm{Nu}})$ for understanding better the effect of temperature modulation on heat transport. A representative time interval that allows a clear comprehension of the modulation effect needs to be chosen. The interval $(0$, $2 \pi$ ) seemed an appropriate interval to calculate $\overline{\mathrm{Nu}}$. The time- averaged Nusselt number $\overline{\mathrm{Nu}}$ is defined as

$$
\overline{\mathrm{Nu}}=\frac{1}{2 \pi} \int_{0}^{2 \pi} \mathrm{Nu} d s .
$$

The amplitude $A_{I}(s)$ is obtained numerically and hence $\overline{\mathrm{Nu}}$ is also to be numerically evaluated. An interesting observation that can be made in $I_{1}$, which determines whether the modulation amplifies or 
diminishes the amplitude of convection. A discussion of the results now follows and conclusions are made.

\subsection{At the third order}

At this stage, we have the following relations

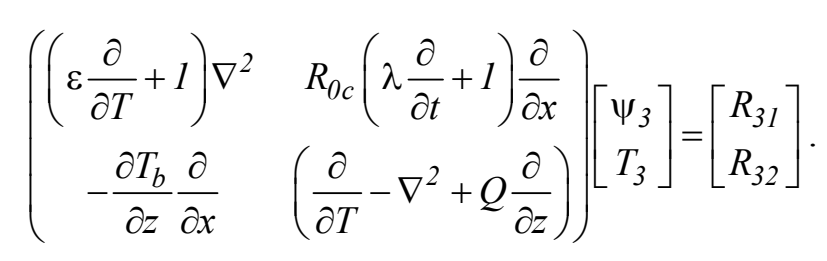

The expressions of $R_{31}$ and $R_{32}$ are given in appendix A1. Now under the solvability condition for the existence of third order solution, we arrive at the following complex Ginzburg Landau equation which describes the temporal variation of the amplitude $A_{l}(s)$ of the convection cell

$$
\frac{\partial A_{l}(s)}{\partial s}-\Upsilon_{l}^{-1} F(s)+\Upsilon_{l}^{-1} k_{l}\left|A_{l}(s)\right|^{2} A_{l}(s)=0 .
$$

Writing $A_{I}(s)$ in the phase-amplitude form, we get

$$
A_{I}(s)\left|A_{l}(s)\right| e^{i \varphi} .
$$

Now substituting the expression Eq.(3.19) in Eq.(3.18), we get the following equation for the amplitude $\left|A_{l}(s)\right|$

$$
\begin{aligned}
& \frac{\partial\left|A_{l}(s)\right|^{2}}{\partial s}=2 p_{r}\left|A_{l}(s)\right|^{2}-2 l_{r}\left|A_{l}(s)\right|^{4}, \\
& \frac{\partial\left(p h\left(A_{l}(s)\right)\right)}{\partial s}=p_{i}-l_{i}\left|A_{l}(s)\right|^{2}
\end{aligned}
$$

where $\gamma 1-1 F(s)=p_{r}+i p_{i}, \gamma 1-1 k_{l}=l_{r}+i l_{i}$ and $p h($.$) represents the phase shift. One can observe here from$ Eq.(3.18) for the case $l_{r}>0$ and $\mathrm{Ra}>\mathrm{Ra}_{c}$ i.e., $p_{r}>0$, the solution given as $A_{l} \sim A_{0} e^{p_{r} s}$ as $s \rightarrow-\infty$ and $A_{l} \rightarrow 0$ is an unstable solution, and a new stable solution develops $A_{l}=\sqrt{\frac{p_{r}}{l_{r}}}$ as $s \rightarrow \infty$, whatever the value of $A_{0}$. This is called super critical pitchfork bifurcation, the base system being linearly unstable for $\operatorname{Ra}>\operatorname{Ra}_{c}$ but settling down as a new laminar flow. The steady state amplitude exists when $\mathrm{Ra}_{c}$ takes positive values.

\section{Results and discussions}

In this paper, we study the combined effect of temperature modulation and vertical through-flow on the Bénard-Darcy oscillatory convection in a viscoelastic fluid saturated porous medium. A weakly nonlinear stability analysis has been performed to investigate the effect of temperature modulation and vertical throughflow on heat transport. The effect of temperature modulation on the Bénard-Darcy system has been assumed to be of order $O\left(\chi^{2}\right)$. This means we consider only small amplitude temperature modulation. Such an assumption will help us in obtaining the amplitude equation of convection in a simple manner and it is 
much easier to obtain it than in the case of the Lorenz model. The purpose of the weak nonlinear theory is to study heat transfer. External regulation of convection is important in the study of convection in porous media. The objective of this article is to consider such, temperature modulations and vertical throughflows for either enhancing or inhibiting the convective heat transfer as is required by a real application. The problem is of interest because of the possibility of controlling the convective instability by adjustment of the transverse throughflow.

Since the porous medium is assumed to be closely packed, the Darcy-model is considered in the governing equation. In order to illustrate the effects of relaxational parameters, $\lambda$, $\varepsilon$, the Péclet number Pe, the frequency $\Omega$ and the amplitude $\delta$ of modulation on heat transport, we plot the curves of the Nusselt number against time. It is observed that Eq.(3.9) leads to an interesting result; i.e., for a horizontal porous layer heated underneath; the oscillatory type of instability is possible only when the relaxation parameter $\lambda$ is greater than the retardation parameter $\varepsilon$. The values of $\delta$ are considered very small, between 0 and 0.1 , since we are studying the effect of small amplitude modulation on the heat transport. Also, since the effect of low frequencies is maximum on the onset of convection as well as on the heat transport, therefore the modulation of temperature is assumed to be of low frequency. A small amount of throughflow in a particular direction either to destabilizes or stabilize the system, considering free or permeable boundaries, hence the values of $\mathrm{Pe}$ are taken around one. The numerical results for $\mathrm{Nu}$ obtained from the expression in Eq.(3.15) by solving the amplitude Eq.(3.18) have been presented in Figs 2 - 8. It is clear to see the expression Eq.(3.12) in conjunction with Eq.(3.18) that $\mathrm{Nu}$ is a function of system parameters. The effect of each type of modulation on heat transport is shown in Figs $2-8$, wherein the plots of the Nusselt number $\mathrm{Nu}$ against $s$ are presented. It is found from the figures that the value of $\mathrm{Nu}$ starts with one and remains constant for quite some time, thus showing the conduction state initially. Then the value of $\mathrm{Nu}$ increases with time, thus showing the conduction state and finally becomes constant on further increasing $s$ thus achieving the steady state.

In Figs 2a-c it is observed that, the direction of throughflow (Pe) does not change the critical Rayleigh number and an increase in the throughflow strength makes the system more stable . This may be due to the fact that throughflow confines significant thermal gradients to a thermal boundary layer at the boundary towards which throughflow is directed. The effective length scale is thus smaller than the porous layer thickness $d$. Hence, the Rayleigh number will be much smaller than the actual value of the critical Rayleigh number. Therefore, higher values of the Rayleigh number are needed for the onset of oscillatory convection as the throughflow strength increases. Gershuni and Zhukhovitskii (1976) wrote that the stabilizing effect may be explained as follows. With an increasing throughflow velocity a temperature boundary layer forms at one of the boundaries. This decreases the effective thickness of the stratified layer of the fluid. On the other hand, the characteristic temperature difference across the porous medium remains fixed. The result was obtained by Khalili and Shivakumar [51] in the case of free-free boundaries. These results are comparable with the results which were obtained by Jones and Persichetti [45], Nield [46] and Shivakumara [49] for porous and fluid layers.

According to Shivakumara and Sureshkumar [53] this effect may be due to the distortion of the steady-state basic temperature distribution from linear to nonlinear by throughflow. A measure of this is given by the basic state temperature and this can be interpreted as a rate of energy transfer into the disturbance by interaction of the perturbation convective motion with the basic temperature gradient. The maximum temperature occurs at a place where the perturbed vertical velocity is high and this leads to an increase in energy supply for destabilization. Figures $2 \mathrm{a}$ and $2 \mathrm{~d}$, show the effect of the time relaxation parameter $\lambda$ on the oscillatory critical Rayleigh number for a fixed value of other governing parameters.

The critical Rayleigh number for the onset of oscillatory convection decreases with increasing the value of $\lambda$, indicating that the effect of increasing $\lambda$ is to advance the onset of oscillatory convection. An opposite effect can be seen in the case of the time retardation parameter $\varepsilon$, given in Figs $2 \mathrm{~b}$ and $2 \mathrm{e}$. Thus, an increase in the value of $\lambda$ and decrease in the value of $\varepsilon$ is to decrease the critical oscillatory Rayleigh number and hence their effect is to promote instability. This may be due to the fact that the relaxation time reduces the shear rate (i.e., increases the elasticity of a viscoelastic fluid) thus causing instability. These results conform to the results obtained by Bhadauria and Kiran [29-30, 57-58]. 

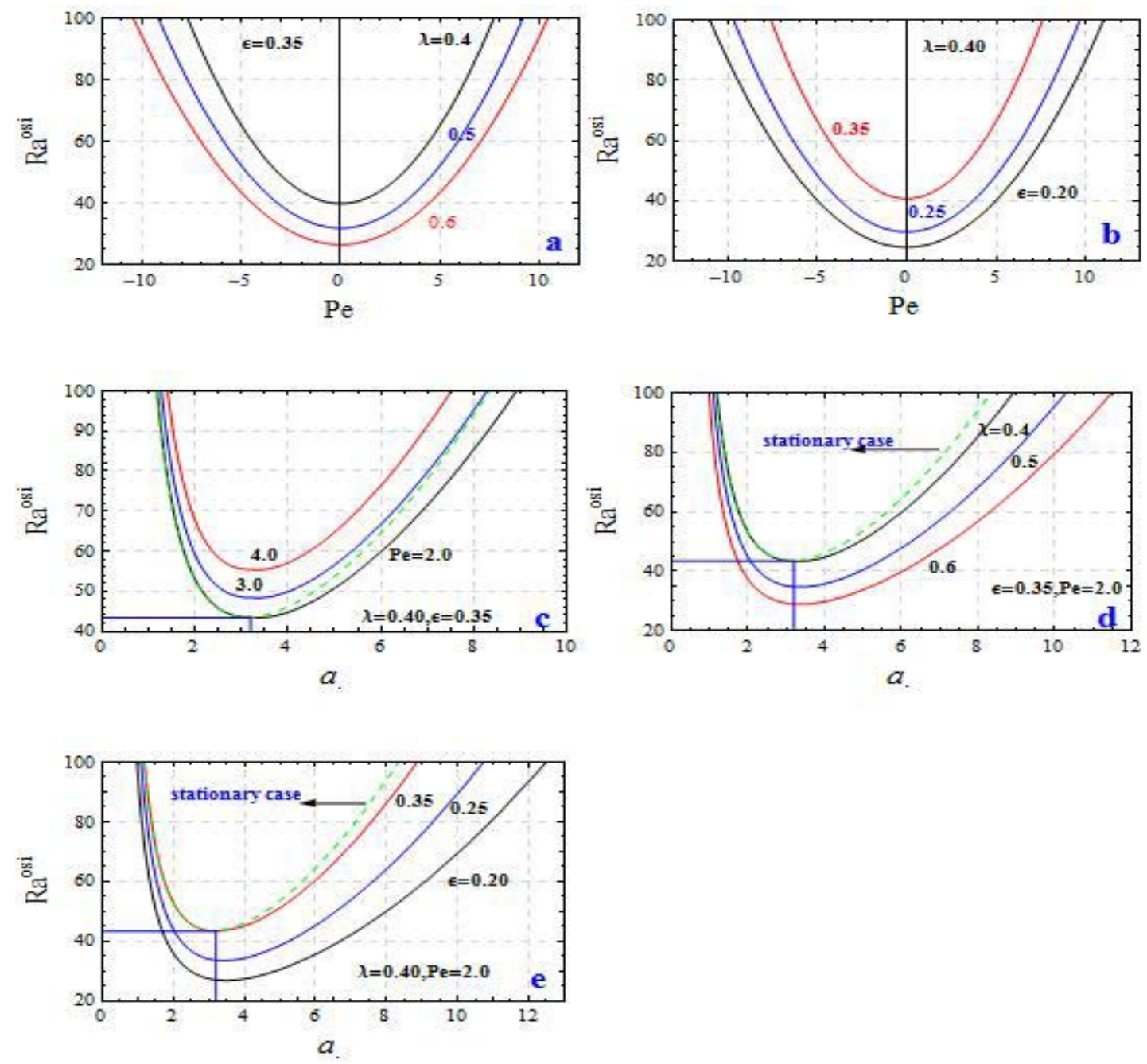

Fig.2. Variation of oscillatory Rayleigh number with different values of Pe and $(\lambda, \in)$.

For IPM case the results are presented in Figs $3 \mathrm{a}-\mathrm{d}$. It is observed that the Nusselt number given by Eq.(3.15) is an odd functions of the Péclet number Pe. So the direction of throughflow influences the heat transfer in the system. From Fig.3a and Fig.3b we note that the direction of throughflow alters the Nusselt number $\mathrm{Nu}$. That is, only upward flow $(\mathrm{Pe}>0$ ) clearly enhances the heat transfer while downward flow $(\mathrm{Pe}<0)$ has reverse effect of diminishing heat transfer of the system. Moreover, the downward flow is more stabilizing as compared to the upward flow. These results are similar to those observed in the case of Newtonian fluids (stationary mode $(\omega=0)$ ) given in Fig.3c. The destabilization may be due to the distortion of the steady-state basic temperature distribution from linear to nonlinear by the throughflow.

A measure of this is given by the term $\frac{d T_{b}}{d z}$ and can be interpreted as a rate of transfer of energy into the disturbance by interaction. From Figs $3 \mathrm{~d}$ and $3 \mathrm{e}$ it is found that the effect of increasing the value of relaxation parameter $\lambda$ is to increase the heat transfer and for retardation parameter is to decrease the heat transfer. These are the common results for most of the viscoelastic fluids obeys, and compatible with the results of Kim et al. [32], Rajib and Layek (2012) and Bhadauria and Kiran [29-30, 57-58]. Further, the amplitude $\delta$ and the frequency of modulation $\Omega$ both have negligible effects on heat transport in this case therefore, for simplicity we have excluded the figure conforms the results of Bhadauria and Kiran [28, 29-30, 57-58]. This IPM case is considered as unmodulated system where 
stability problem (Davis [5]) admits no time periodic disturbances so that tuning frequency and varying amplitude is not apparent. These results for viscoelastic fluids are the same as unmodulated system obtained by Malashetty and Swamy [39], Kumar and Bhadauria [40-41], Rajib and Layek [64] and Reza and Gupta [56].
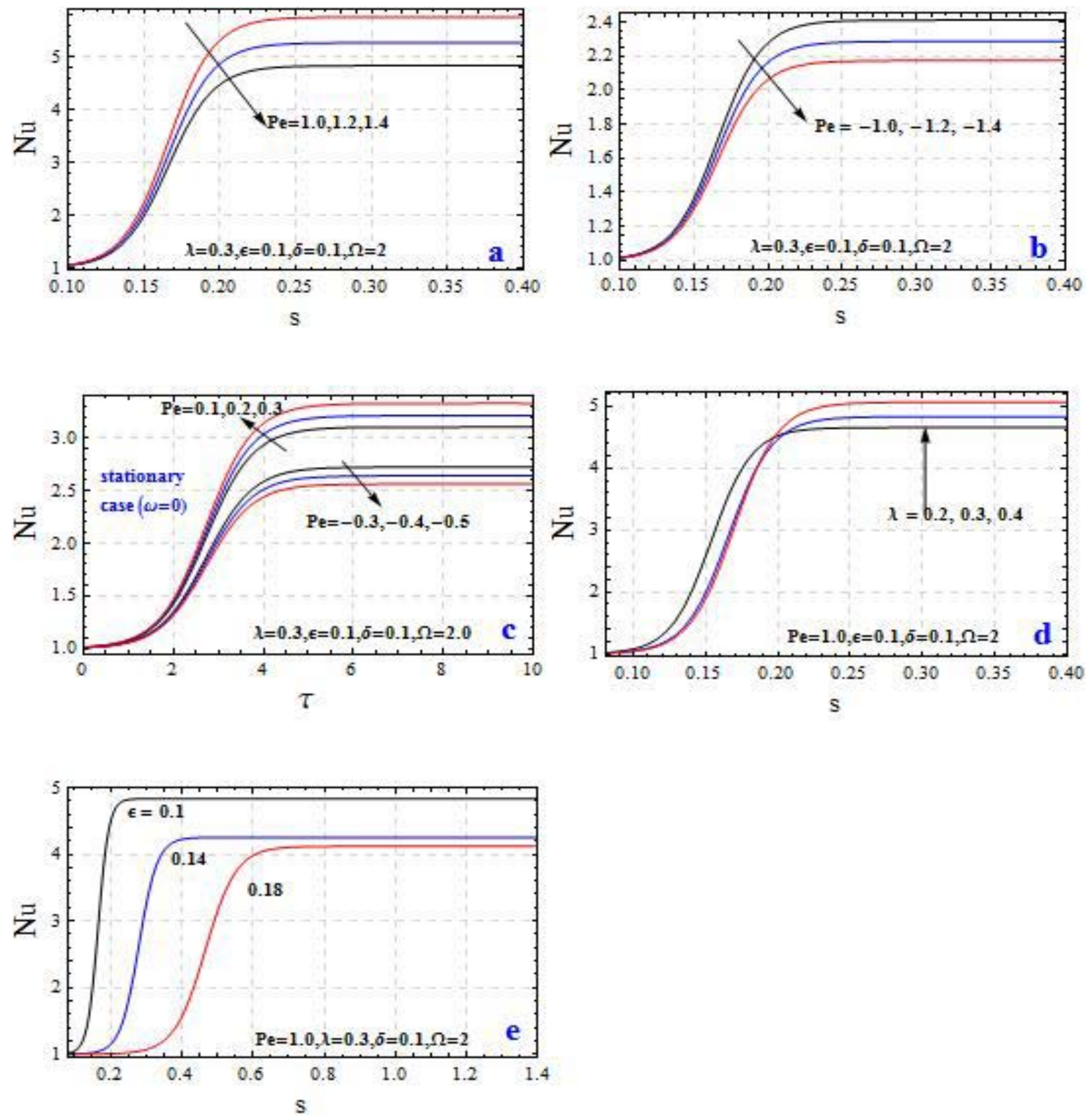

Fig.3. Effect of various parameters on heat transfer for in - phase modulation (a) Pe (b) -Pe (c) $\lambda$ (d) $\in$.

In Figs 4a-f, we have depicted the variation of $\mathrm{Nu}$ with slow time scale $s$ for out of phase modulations. It is found that $\mathrm{Nu}$ starts with one, increases with increasing time $s$ and then becomes oscillatory. From these figures one can observe that the oscillatory nature of the graphs due to frequency $\Omega$ of modulation only but not of oscillatory frequency $\omega$. Finally these frequencies never interact each other and they are entirely different each other. We observe from the Figs $4 \mathrm{a}$-d that the effect of Pe, $\lambda, \varepsilon$ on heat transport is found to be similar to those of IPM. Further, we found in Fig.4e that the effect of amplitude of modulation is to increase the magnitude of $\mathrm{Nu}$, thus increasing the heat transport, and thus 
advancing the convection. Also, from Fig.4f, we observe that increasing in frequency of modulation $\Omega$ decreases the magnitude of $\mathrm{Nu}$, and so the effect of frequency of modulation is to diminish the heat transport. At high frequency the effect of temperature modulation on thermal instability disappears altogether. This modulation result agrees quite well with the linear theory results Venezian [6] and Finucane and Kelly [61], where the correction in the critical value of Rayleigh number due to temperature modulation becomes almost zero at high frequencies. Similar results can be seen in Bhadauria and Kiran [28, 29-30, 57-58] for viscoelastic fluid or porous layers in the absence of throughflow. Here, we are not presenting the results for the case of Lower boundary modulation; as we found that the effect of each parameter on the heat transport is qualitatively similar to those obtain in OPM case. However, the magnitude of $\mathrm{Nu}$ in this case is less than that in the case of OPM, thus in Fig.5, a comparison of results of in phase modulation, out of phase modulation and when only lower boundary temperature is modulated, is presented. It is found that OPM and LBMO (modulated system) enhances heat transfer rather than the IPM case (unmodulated system). It is also observed that in Fig.5 lower boundary modulation is just as gravity modulation. Figure 6, shows that the oscillatory mode of convection advances the onset of convection, and hence heat transfer rather than stationary mode of convection. Hence oscillatory mode of convection sets in before stationary mode of convection conforms the results of Bhadauria and Kiran [28, 29-30, 57-58].
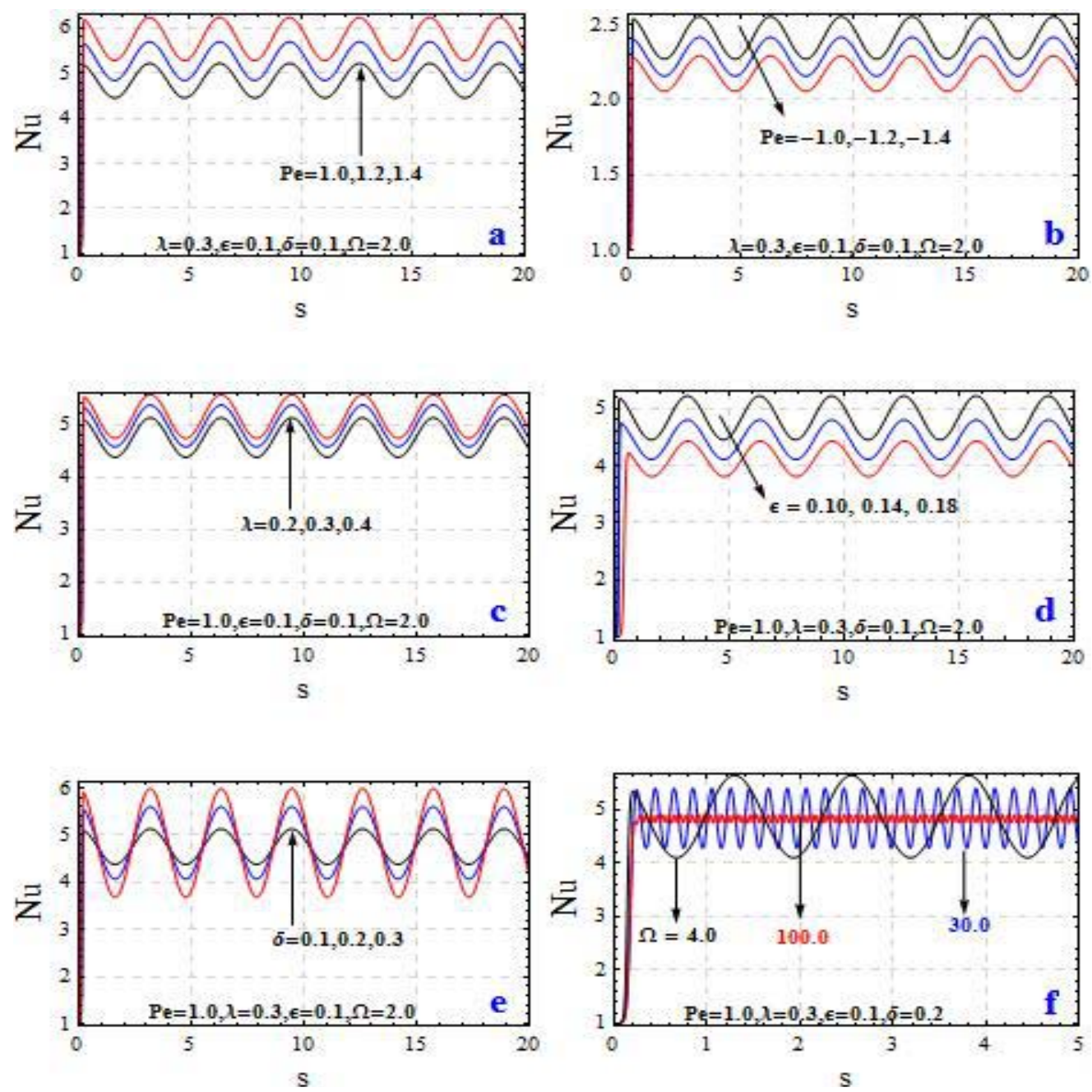

Fig.4. Effect of various parameters on heat transfer for out of phase modulation (a) Pe (b) -Pe (c) $\lambda$ (d) $\in(\mathrm{e}) \delta$ (f) $\Omega$. 


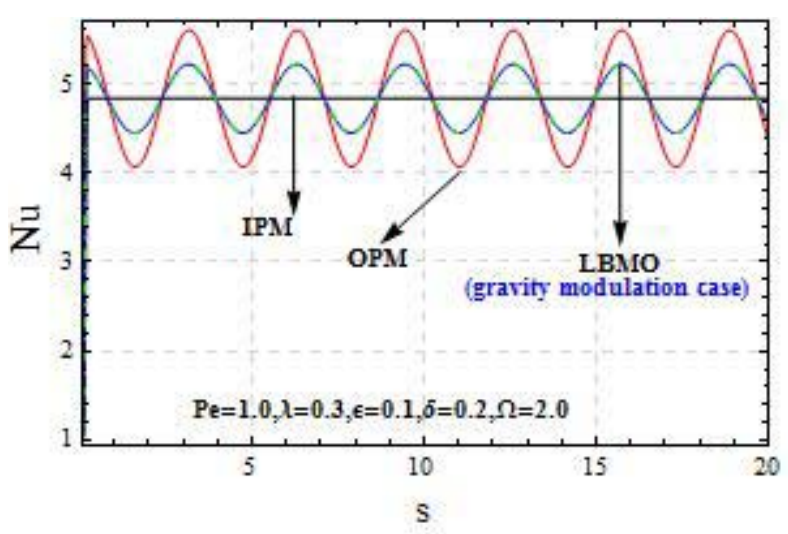

Fig.5. Comparision between various cases of modulation.

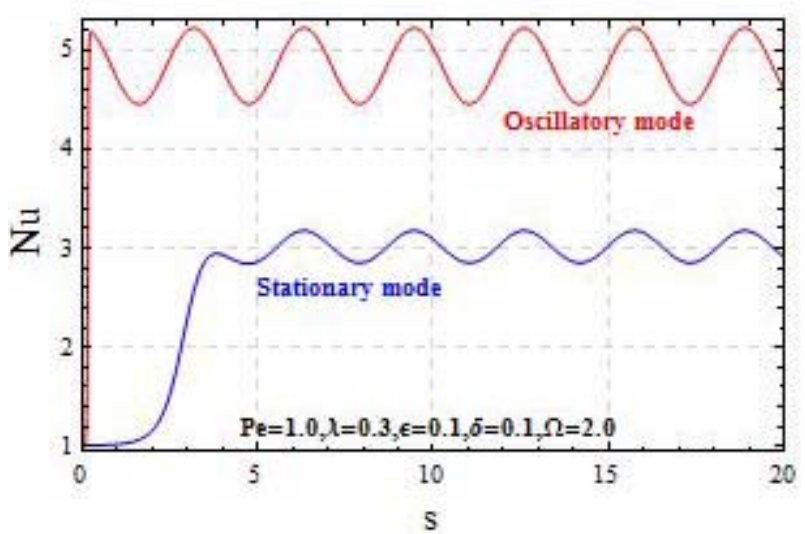

Fig.6. Comparision between stationary and oscillatory cases.

For better understanding of temperature modulation Figs 7 and 8 drawn while considering phase angle $\theta$ according to Siddheshwar et al. [65] and Bhadauria and Kiran [29-30] the effect of modulation on mean Nusselt number $\mathrm{Nu}$ depends on both the phase difference $\theta$ and frequency $\Omega$ of thermal modulation than only on the choice of the small amplitude modulation. From the Figs 7 and 8 it is shown that for a given value of $\Omega$ there is a range of $\theta$ in which $\mathrm{Nu}$ increases with increasing $\theta$ and another range in which $\mathrm{Nu}$ decreases. Thus, it is concluded that, the suitable combination of choices of $\Omega$ and $\theta$ can be made depending on the demands on heat transport applications. Hence heat transfer can be regulated (enhanced or reduced) with the external temperature modulation effectively. These are the results compatible with the results of Malashetty et al. [66]. We also can observe our results in Figs 7-8 are the results which are similar to Siddheshwar et al. [65] for the Newtonian fluid case Bhadauria and Kiran [29-30] for non-Newtonisn fluids. It is clear that for temperature modulation the boundary temperatures should not be in in-phase modulation (synchronized), where the effect of modulation is negligible on heat transport. 

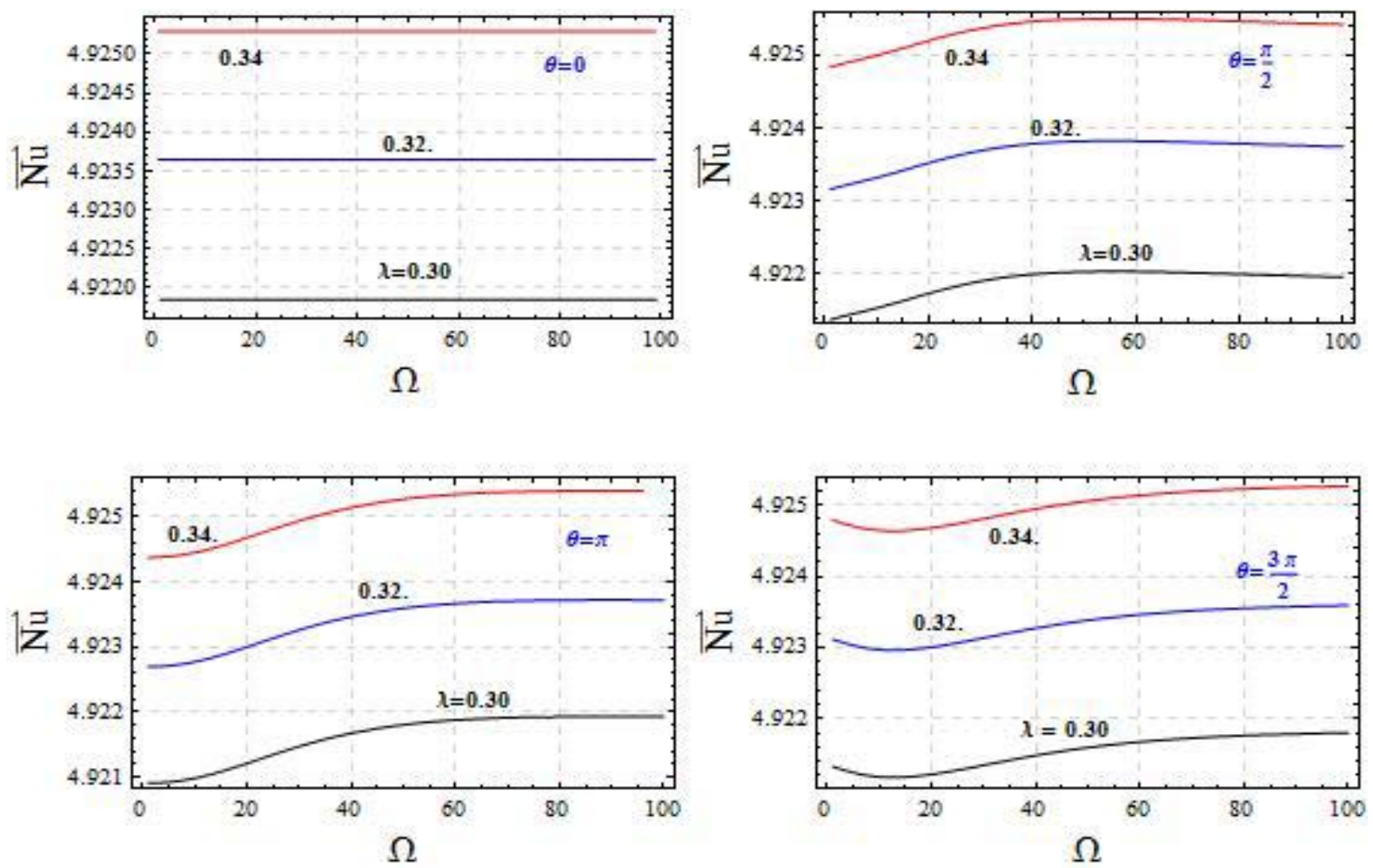

Fig.7. Effect of frequency of modulation on $\overline{\mathrm{Nu}}$ for various values of $\theta$ and $\lambda$.
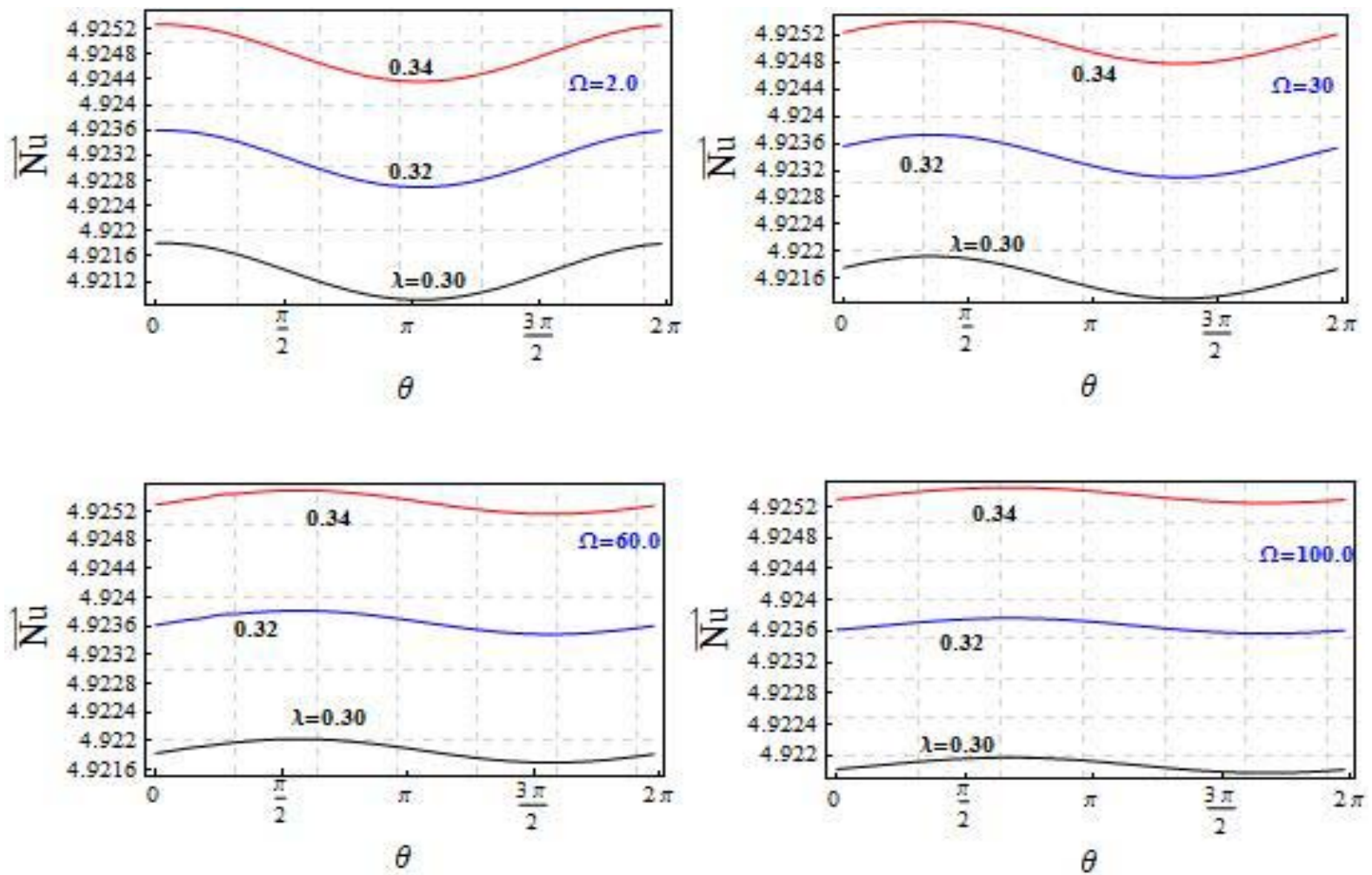

Fig.8. Effect of $\theta$ on $\overline{\mathrm{Nu}}$ for various values of $\Omega$ and $\lambda$. 


\section{Conclusions}

We have analyzed the effect of temperature modulation and vertical throughflow on a viscoelastic fluid saturated porous medium by performing a weakly nonlinear stability analysis resulting in the complex form of Ginzburg-Landau amplitude equation. The following conclusions are drawn from the above analysis:

1. Throughflow has a stabilizing effect on the onset of convection irrespective of its direction.

2. The effect of upward throughflow enhances heat transfer.

3. The effect of in-phase modulation is negligible on heat transport in the system.

4. The effect of $\lambda$ is to enhance heat transport for all three types of modulations.

5. The effect of $\varepsilon$ is to decrease heat transport for all three types of modulations.

6. In the case of IPM, Nu increases steadily for intermediate values of time $s$ and ultimately becomes constant when $s$ is large.

7. In the case of OPM and LBMO, Nu shows an oscillatory nature.

8. Supercritical pitchfork bifurcation exits for Eq.(3.18).

9. Lower boundary modulation results are same as gravity modulation results.

\section{Acknowledgement}

The author Dr. Palle Kiran is grateful to the Department of Atomic Energy, Government of India, for providing financial assistance in the form of NBHM-Post-doctoral Fellowship (Lett. No:2/40(27)/2015/R\&D-II/401). The authors are grateful to the unknown referees for their useful comments that helped to refine the paper in its current form.

\section{Nomenclature}

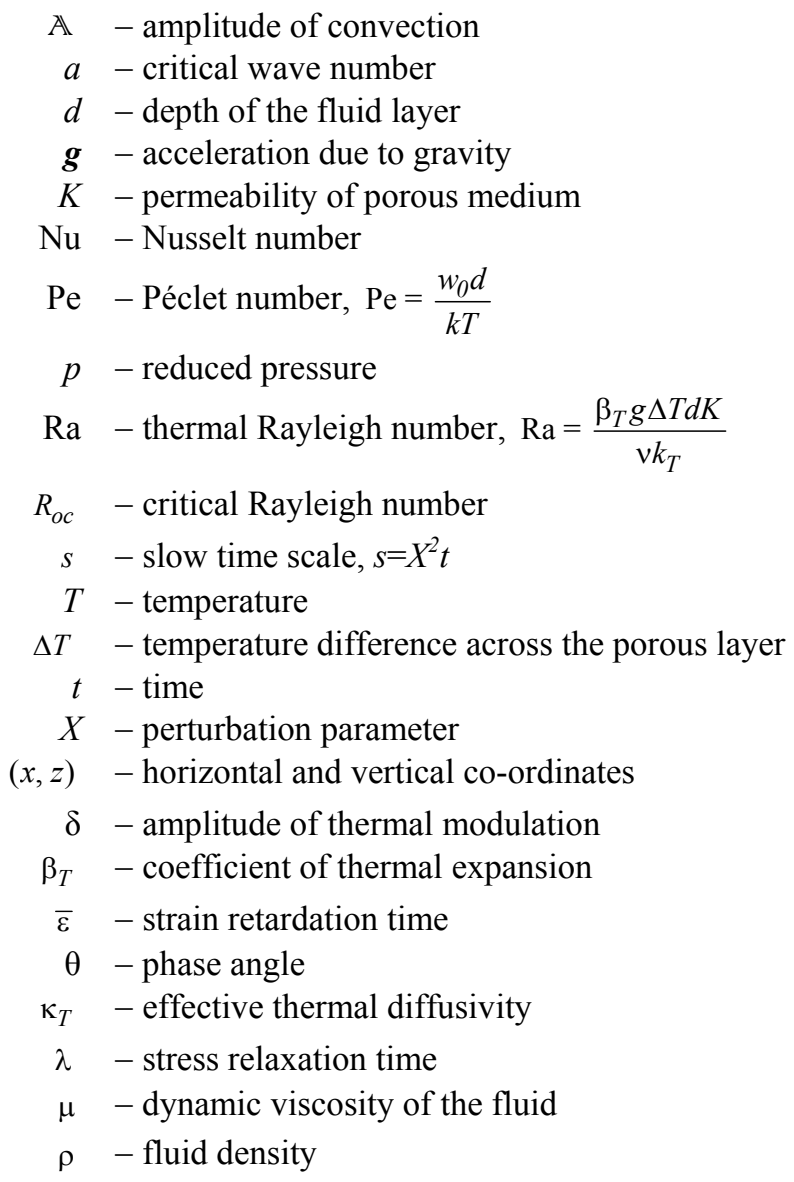




$$
\begin{aligned}
v & - \text { kinematic viscosity, } \frac{\partial^{2}}{\partial x^{2}}+\frac{\partial^{2}}{\partial y^{2}}+\frac{\partial^{2}}{\partial z^{2}}\left(\frac{\mu}{\rho_{0}}\right) \\
\phi & - \text { porosity } \\
\psi & - \text { stream function } \\
\Omega & - \text { frequency of modulation } \\
\omega & - \text { dimensionless oscillatory frequency } \\
\nabla^{2} & -\frac{\partial^{2}}{\partial x^{2}}+\frac{\partial^{2}}{\partial y^{2}}+\frac{\partial^{2}}{\partial z^{2}}
\end{aligned}
$$

\section{Subscripts}

$$
\begin{array}{ll}
b & - \text { basic state } \\
c & - \text { critical } \\
0 & - \text { reference value }
\end{array}
$$

\section{Superscripts}

$$
\begin{aligned}
& * \quad \text { - dimensionless quantity } \\
& \text { - perturbed quantity }
\end{aligned}
$$

\section{Appendix A1}

The coefficients given in Eq.(2.17) are $f^{\prime}=\frac{\mathrm{Pe} e^{P e z}}{1-e^{\mathrm{Pe}}}, f_{1}^{\prime}(z, t)=\left[B\left(\theta_{2}\right) e^{\theta_{2 z}}+B\left(-\theta_{2}\right) e^{-\theta_{2 z}}\right] e^{-i \Omega t}$ $B\left(\theta_{2}\right)=\frac{\theta_{1}+\theta_{2}}{2} \frac{\left(e^{-i \varphi}-e^{\theta_{1}-\theta_{2}}\right)}{e^{\theta_{1}}\left(e^{\theta_{2}}-e^{-\theta_{2}}\right)}, \theta_{1}=\frac{\mathrm{Pe}}{2}, \theta_{1}=\frac{\sqrt{\mathrm{Pe}^{2}+4 \lambda^{2}}}{2}$ and $\lambda=(1-i) \sqrt{\frac{\Omega}{2}}$.

The coefficients given in Eq.(3.17) are

$$
\begin{aligned}
& R_{31}=-\varepsilon \frac{\partial}{\partial s}\left(\nabla^{2} \psi_{1}\right)-R_{0 c} \lambda \frac{\partial}{\partial s}\left(\frac{\partial T_{1}}{\partial x}\right)-R_{2}\left(\lambda \frac{\partial}{\partial t}+1\right)\left(\frac{\partial T_{1}}{\partial x}\right), \\
& R_{32}=\frac{\partial \psi_{1}}{\partial x}\left(\frac{\partial T_{2}}{\partial z}\right)+\delta f_{2}(z, s) \frac{\partial \psi_{1}}{\partial x}-\left(\frac{\partial T_{1}}{\partial s}\right) .
\end{aligned}
$$

The coefficients given in Eq.(3.18) are

$$
\begin{aligned}
& \Upsilon_{1}=\left[\varepsilon c-\frac{4 \pi^{2} a^{2} \lambda R_{0 c}}{(c+i \omega)\left(4 \pi^{2}+\mathrm{Pe}^{2}\right)}-\frac{4 \pi^{2} a^{2} R_{0 c}(1+i \omega \lambda)}{(c+i \omega)^{2}\left(4 \pi^{2}+\mathrm{Pe}^{2}\right)}\right], \\
& F(S)=\left[\frac{4 \pi^{2} a^{2} R_{0 c}(1+i \omega \lambda)}{(c+i \omega)\left(4 \pi^{2}+\mathrm{Pe}^{2}\right)}-\frac{2 a^{2} R_{0 c}(1+i \omega \lambda)}{(c+i \omega)^{2}} \delta I_{1}\right], \\
& I_{1}=\int_{0}^{1} f_{2}(z, s) \sin ^{2}(\pi z) d z, \quad I_{1}=\int_{0}^{1} f_{2}(z, s) \sin ^{2}(\pi z) d z .
\end{aligned}
$$




\section{References}

[1] Ingham D.B. and Pop I. (1998): Transport Phenomena in Porous Media. - Oxford: Pergamon.

[2] Nield D.A. and Bejan A. (2012): Convection in Porous Media 4th edn. - New York: Springer.

[3] Vafai K. (2000): Handbook of Porous Media. - New York: Dekker.

[4] Vafai K. (2005): Handbook of Porous Media 2nd edn. - Boca Raton: CRC Press.

[5] Davis S. (1976): The stability of time-periodic flows. - Annual Review of Fluid Mech., vol.8, pp.57-74.

[6] Venezian G. (1969): Effect of modulation on the onset of thermal convection. - J. Fluid Mech., vol.35, pp.243-254.

[7] Caltagirone J.P. (1976): Stabilite dune couche poreuse horizontale soumise a des conditions aux limites periodiques. - Int. J. Heat Mass Transfer, vol.19, pp.815-820.

[8] Chhuon B. and Caltagirone J.P. (1979): Stability of a horizontal porous layer with time-wise periodic boundary conditions. - Trans. ASME, J. Heat Transfer, vol.101, pp.244-248.

[9] Rudraiah N., Veerappa B. and Balachandra Rao S. (1980): Effects of non-uniform thermal gradient and adiabatic boundaries on convection in porous media. - ASME, J. Heat Transfer, vol.102, pp.254-260.

[10] Rudraiah N., Veerappa B. and Balachandra Rao S. (1982): Convection in a fluid saturated porous layer with non-uniform temperature gradient. - Int. J. Heat Mass Transfer, vol.25, pp.1147-1156.

[11] Rudraiah N. and Malashetty M.S. (1990): Effect of modulation on the onset of convection in a sparsely packed porous layer. - Trans. ASME, J. Heat Transfer, vol.112, pp.685-689.

[12] Malashetty M.S. and Wadi V.S. (1999): Rayleigh-Bénard convection subject to time dependent wall temperature in a fluid saturated porous layer. - Fluid Dyn. Res. vol.24, pp.293-308.

[13] Bhatia P.K. and Bhadauria B.S. (2000): Effect of modulation on thermal convection instability. - Z. Naturforsch, vol.55a, pp.957-966.

[14] Bhati, P.K. and Bhadauria B.S. (2001): Effect of low-frequency modulation on thermal convection instability. - Z. Naturforsch, vol.56a, pp.507-522.

[15] Bhadauria B.S. and Bhatia P.K. (2002): Time-periodic heating of Rayleigh-Bénard convection. - Physica Scripta. vol.66, No.1, pp.59-65.

[16] Bhadauria B.S. (2007a): Thermal modulation of Rayleigh Bénard convection in a sparsely packed porous medium. - J. Porous Media, vol.10, pp.175-188.

[17] Bhadauria B.S. (2007b): Fluid convection in a rotating porous layer under modulated temperature on the boundaries. - Transp. Porous Media, vol.67, No.2, pp.297-315.

[18] Bhadauria B.S. (2007c): Magnetofluidconvection in a rotating porous layer under modulated temperature on the boundaries. - ASME J. Heat Transfer, vol.129, pp.835-843.

[19] Bhadauria B.S. (2007d): Double diffusive convection in a rotating porous layer with modulated temperature on the boundaries. - J. Porous Media, vol.10, No.6, pp.569-584.

[20] Bhadauria B.S. (2007e): Double diffusive convection in a porous medium with modulated temperature on the boundaries. - Transp. Porous Media, vol.70, No.2, pp.191-211.

[21] Bhadauria B.S. (2008a): Combined effect of temperature modulation and magnetic field on the onset of convection in an electrically conducting-fluid-saturated porous medium. - ASME J. Heat Transfer, vol.130, No.5, pp.0526(19).

[22] Bhadauria B.S. (2008b): Effect of temperature modulation on Darcy convection in a rotating porous medium. - J. Porous Media, vol.11, No.4, pp.361-375.

[23] Bhadauria B.S. and Sherani A. (2008a): Onset of Darcy-convection in a magnetic-fluid-saturated porous medium subjected to temperature modulation of the boundaries. - Transp. Porous Media, vol.73, pp.349-368. 
[24] Bhadauria B.S. and Sherani A. (2008b): Thermal modulation of double diffusive convection in a porous medium. - Z. Naturforsch., vol.63a, pp.291-300.

[25] Bhadauria B.S. and Suthar O.P. (2009): Effect of thermal modulation on the onset of centrifugally driven convection in a vertical rotating porous layer placed far away from the axis of rotation. - J. Porous Media, vol.12, No.3, pp.239-252.

[26] Bhadauria B.S. and Srivastava K. (2010): Magneto-double diffusive convection in an electrically conductingfluidsaturated porous medium with temperature modulation of the boundaries. - Int. J. Heat Mass Transfer, vol.53, pp.2530-2538.

[27] Bhadauria B.S., Hashim I. and Siddheshwar P.G. (2013): Effects of time-periodic thermal boundary conditions and internal heating on heat transport in a porous medium. - Transp. Porous Media, vol.97, pp.185-200.

[28] Bhadauria B.S. and Kiran P. (2013): Heat transport in an anisotropic porous medium saturated with variable viscosity liquid under temperature modulation. - Transp. Porous Media, vol.100, pp.279-295.

[29] Bhadauria B.S. and Kiran P. (2014a): Weakly nonlinear oscillatory convection in a viscoelastic fluid saturating porous medium under temperature modulation. - Int. J. Heat Mass Transfer, vol.77, pp.843-851.

[30] Bhadauria B.S. and Kiran P. (2014b): Heat and mass transfer for oscillatory convection in a binary viscoelastic fluid layer subjected to temperature modulation at the boundaries. - Int. Communi in Heat and Mass Transfer, vol.58, pp.166-175.

[31] Rudraiah N., Kaloni P.N. and Radhadevi P.V. (1989): Oscillatory convection in a viscoelastic fluid through a porous layer heated from below. - Rheol. Acta, vol.28, pp.48-53.

[32] Kim M.C., Lee S.B., Kim S. and Chung B.J. (2003): Thermal instability of viscoelastic fluids in porous media. - Int. J. Heat Mass Transfer, vol.46, pp.5065-5072.

[33] Yoon D.Y., Kim M.C. and Choi C.K. (2004): The onset of oscillatory convection in a horizontal porous layer saturated with viscoelastic liquid. - Transp. Porous Media, vol.55, pp.275-284.

[34] Malashetty M.S., Shivakumara I.S., Sridhar K. and Mahantesh S. (2006): Convective instability of Oldroyd-B fluid saturated porous layer heated from below using a thermal non-equilibrium model. - Transp. Porous Media, vol.64, pp.123-139.

[35] Bertola V. and Cafaro E. (2006): Thermal instability of viscoelastic fluids in horizontal porous layers as initial problem. - Int. J. Heat Mass Transfer, vol.49, p p.4003-40012.

[36] Sheu L.J., Tam L.M., Chen J.H., Chen H.K., Lin K.T and Kang Y. (2008): Chaotic convection of viscoelastic fluids in porous media. - Chaos, Solitons Fractals, vol.37, pp.113-124.

[37] Sheu L.J., Chen J.H, Chen H.K., Tam L.M. and Chao Y.C. (2009): A unified system describing dynamics of chaotic convection. - Chaos, Solitons Fractals, vol.41, pp.123-130.

[38] Wang S.W. and Tan W.C. (2008): Stability analysis of double-diffusive convection of Maxwell fluid in a porous medium heated from below. - Phys. Lett. A, vol.372, pp.3046-3050.

[39] Malashetty M.S., Swamy M. and Heera R. (2009): The onset of convection in a binary viscoelastic fluid saturated porous layer. - Z. Angew. Math. Mech., vol.89, pp.356-369.

[40] Kumar A. and Bhadauria B.S. (2011a): Double diffusive convection in a porous layer saturated with viscoelastic fluid using a thermal non-equilibrium model. - Phys. Fluids, vol.23, pp.054101.

[41] Kumar A. and Bhadauria B.S. (2011b): Nonlinear two dimensional double diffusive convection in a rotating porous layer saturated by a viscoelastic fluid. - Transp. Porous Media, vol.87, pp.229-250.

[42] Wooding R.A. (1960): Rayleigh instability of a thermal boundary layer in flow through aporous medium. - J. Fluid Mech., vol.9, pp.183-192.

[43] Sutton F.M. (1970): Onset of convection in a porous channel with net throughflow. - Phys. Fluids, vol.13, pp.1931.

[44] Homsy G.M. and Sherwood A.E. (1976): Convective instabilities in porous media with throughflow. AIChE J., vol.22, pp.168-174. 
[45] Jones M.C. and Persichetti J.M. (1986): Convective instability in packed beds with throughflow. - AIChE J., vol.32, pp.1555-1557.

[46] Nield D.A. (1987): Convective instability in porous media with throughflow. - AIChE J., vol.33, pp.1222-1224.

[47] Shivakumara I.S. (1997): Effects of throughflow on convection in porous media. - Proc. 7th Asian Congr. Fluid Mechanics, vol.2, pp.557-560.

[48] Khalili A. and Shivakumara I.S. (1998): Onset of convection in a porous layer with net throughflow and internal heat generation. - Phys. Fluids, vol.10, pp.315.

[49] Shivakumara I.S. (1999): Boundary and inertia effects on convection in a porous media with throughflow. Acta Mech., vol.137, pp.151-165.

[50] Shivakumara I.S. and Khalili A. (2001): On the stability of double diffusive convection in a porous layer with throughflow. - Acta Mech., vol.152, pp.165-175.

[51] Khalili A. and Shivakumara I.S. (2003): Non-Darcian effects on the onset of convection in a porous layer with throughflow. - Transp. Porous Media, vol.53, pp.245-263.

[52] Shivakumara I.S. and Nanjundappa C.E. (2006): Effects of quadratic drag and throughflow on double diffusive convection in a porous layer. - Int. Communi. Heat and Mass Transfer, vol.33, pp.357-363.

[53] Shivakumara I.S. and Sureshkumar S. (2007): Convective instabilities in a viscoelastic fluid saturated porous medium with throughflow. - J. Geophys. Eng., vol.4, pp.104-115.

[54] Brevdo L. (2009): Three-dimensional absolute and convective instabilities at the onset of convection in a porous medium with inclined temperature gradient and vertical throughflow. - J. Fluid Mech., vol.641, pp.475-487.

[55] Barletta A., di Schio E.R. and Storesletten L. (2010): Convective roll instabilities of vertical throughflow with viscous dissipation in a horizontal porous layer. - Transp. Porous Media, vol.81, pp.461-477.

[56] Reza M. and Gupta A.S. (2012): Magnetohydrodynamics thermal instability in a conducting fluid layer with through flow. - Int. J. Non-Linear Mech., vol.47, pp.616-625.

[57] Bhadauria B.S. and Kiran P. (2014c): Weak nonlinear oscillatory convection in a viscoelastic fluid saturated porous medium under gravity modulation. - Transp. in Porous Media, vol.104, No.3, pp.451-467.

[58] Bhadauria B.S. and Kiran P. (2014d): Weak nonlinear oscillatory convection in a viscoelastic fluid layer under gravity modulation. - Int. J. Non-linear Mech., vol.65, pp.133-140.

[59] Alishaev M.G. and Mirzadjanzade A.Kh. (1975): For the calculation of delay phenomenon in filtration theory. - Izvestiya Vuzov Neft i Gaz, vol.6, pp.71-74.

[60] Rosenblat S. (1986): Thermal convection of a viscoelastic fluid. - J. Non-Newtonian Fluid Mech., vol.21, pp.201-223.

[61] Finucane R.G. and Kelly R.E. (1976): Onset of instability in a fluid layer heated sinusoidally from below. Int. J. Heat Mass Transfer, vol.19, pp.71-85.

[62] Horton C.W. and Rogers F.T. (1945): Convection currents in a porous medium. - J. Appl. Physics, vol.16, pp.367-370.

[63] Lapwood E.R. (1948): Convection of a fluid in a porous medium. - Proc. Camb. Philos. Soc, vol.44, pp.508-521.

[64] Rajib B. and Layek G.C. (2012): The onset of thermo-convection in a horizontal viscoelastic fluid layer heated underneath. - Thermal Energy Power Eng., vol.1, pp.1-9.

[65] Siddheshwar P.G., Bhadauria B.S. and Suthar Om.P. (2013): Synchronous and asynchronous boundary temperature modulations of Bénard-Darcy convection. - Int. J. Nonlinear Mech., vol.49, pp.84-89.

[66] Malashetty M.S. and Basavaraja D. (2002): Rayleigh Bénard convection subject to time dependent wall temperature/ gravity in a fluid saturated anisotropic porous medium. - Int. J. Heat Mass Transfer, vol.38, pp.551-563. 